\title{
1 Molecular and genetic approaches for assaying human cell type synaptic 2 connectivity
}

Mean-Hwan Kim ${ }^{1 *}$, Cristina Radaelli ${ }^{1 \#}$, Elliot R. Thomsen ${ }^{1 \#, ~ J o s e p h ~ T . ~ M a h o n e y ~}{ }^{1}$, Brian Long ${ }^{1}$, Michael J. Taormina $^{1}$, Sara Kebede ${ }^{1}$, Clare Gamlin ${ }^{1}$, Staci A. Sorensen ${ }^{1}$, Luke Campagnola ${ }^{1}$, Nick Dee ${ }^{1}$, Florence D’Orazi ${ }^{1}$, Andrew L. Ko ${ }^{2}$, Jeffrey G. Ojemann ${ }^{2}$, Daniel L. Silbergeld ${ }^{2}$, Ryder P. Gwinn ${ }^{3}$, Charles Cobbs ${ }^{3}$, C. Dirk Keene ${ }^{4}$, Tim Jarsky ${ }^{1}$, Gabe Murphy ${ }^{1,5}$, Hongkui Zeng ${ }^{1}$, Philip R. Nicovich ${ }^{1}$, Jonathan T. Ting ${ }^{1,5}$, Boaz P. Levi ${ }^{1 *}$, Ed S. Lein $^{1,2}$

1 Allen Institute for Brain Science, Seattle, WA, USA

2 Department of Neurological Surgery, School of Medicine, University of Washington, Seattle, WA, USA

3 Swedish Neuroscience Institute, Seattle, WA, USA

4 Department of Pathology, University of Washington, Seattle, WA, USA

5 Department of Physiology \& Biophysics, University of Washington, Seattle, WA, USA

\# Equal contribution.

*Correspondence should be addressed to M.H.K (meanhwank@ alleninstitute.org) or B.P.L (boazl@alleninstitute.org).

\begin{abstract}
Prospective and post-hoc molecular identification of specific neuron types is essential for functional studies of cellular and synaptic properties. We demonstrate a thick brain slice mFISH technique applied to multipatch-clamp recordings in human cortical slices obtained from neurosurgical-excised tissue to reveal the molecular and morpho-electric properties of synaptically connected neurons, both with and without prospective AAV based genetic labeling. This "quadruple modality" methodology should be extensible to other local brain circuits in many organisms.
\end{abstract}




\section{INTRODUCTION}

Intrinsic membrane properties and synaptic connectivity have been extensively studied in rodent brain slice preparations aided by various genetic tools (Pfeffer et al.,2013; He et al., 2016; Tremblay et al., 2016). Unfortunately, far less is known about cellular and circuit properties of the human brain. Work from several research groups has shown that electrophysiological properties and local connectivity can be studied in acute human neocortical slices derived from surgical resections (Molnar et al., 2008; Jiang et al., 2012; Testa-Silva et al., 2014; Kalmbach et al., 2018; Beaulieu-Laroche et al., 2018; Seeman et al., 2018; Peng et al., 2019). These excised tissues are often distal to epileptic seizure foci or deep brain tumors and in many cases are devoid of overt pathology (Szabadics et al., 2006; Verhoog et al., 2013; Tian et al., 2014; Wang et al., 2015; Beaulieu-Laroche et al., 2018; Berg et al 2020). Human ex vivo neocortical slices can also be cultured for weeks to months (Eugene et al, 2014; Schwarz et al., 2017), and viral transgenesis allows genetic manipulation of cells in brain slices (Andersson et al, 2016; Le Duigou et al, 2018; Ting et al., 2018; Mich et al., 2020; Schwarz et al, 2019).

Here, we present new innovations to study cellular and synaptic physiology at the level of molecularly defined cell subclasses in human ex vivo brain slices. We applied conventional patch-clamp electrophysiology with multiple cells simultaneously (multi-patch-clamp, refer to MPC) to investigate local synaptic connectivity, cellular morphology and intrinsic membrane properties, coupled with post-hoc multiplexed in situ hybridization (mFISH) to reveal molecular properties of characterized neurons and synapses. We also demonstrate the application of cell class-specific adeno-associated virus (AAV) vectors to prospectively mark human cell classes for functional characterization of the neocortical circuitry, where RNA expression, synaptic connectivity, intrinsic physiology, and cell morphology can all be assessed. This quadruple modality approach connects RNA-based molecular profiling of individual neurons with cell morphology and their functional circuit properties including synaptic connectivity and plasticity, demonstrated here in the human cortex but likely broadly applicable to traditionally non-genetically manipulatable organisms.

\section{RESULTS}

The goal of the study was to combine cell subclass-specific marker gene detection with simultaneous MPC recordings to measure local synaptic connectivity, morphology, and intrinsic membrane properties of connected neurons in the human neocortex. Two main experimental applications were performed. One is an acute brain slice preparation and the other is an organotypic brain slice culture preparation. Both acute and cultured brain slice experiments can be performed from a single surgical case given that multiple slices are generated from a single resection whose average volume is $1.39 \pm 0.57 \mathrm{~cm}^{3}$ (mean \pm standard error of mean; averaged over $n=12 \mathrm{cases}$ ). Notably, the time window to perform acute ( $<12$ hours following surgical resection) and slice culture experiments (2.5-7 days in vitro, DIV; Fig. 1 and Extended Data Fig. 1) are different. In slice culture experiments, viral vector and regulatory region confer a cell class or subclass-specific fluorescent label to the tissue and facilitates targeting the marked neurons for MPC recordings. In acute slice preparations, neurons were targeted based on somatic shape as visualized by oblique illumination. After MPC electrophysiology experiments concluded, mFISH was conducted on fixed brain slices, and morphological analyses were performed based on backfilled biocytin/streptavidin staining (Fig. 1). Thus, these experiments provide deep characterization of neocortical microcircuitry by simultaneous measurement of intrinsic membrane properties, synaptic connectivity, marker gene detection, and cell morphology.

\section{Local synaptic connectivity and their intrinsic membrane properties in acute human cortical slices}

With the $350 \mu \mathrm{m}$ thick acute brain slice preparation, connectivity assays with MPC recordings were performed by targeting cell bodies located between 50 and $120 \mu \mathrm{m}$ below the surface of the slice to avoid 
truncation of dendrites and other superficial damage that occurs during slice preparation (Fig. 2a-g; Seeman et al., 2018). For molecular characterization, we opted to apply mFISH in patched slices since measurement of mRNA allows direct correlation with single-cell/single-nucleus RNA-seq data which has been used to classify cortical neurons (Lake et al., 2016; Hodge et al., 2019), and is more easily adapted to new gene targets. Hybridization chain reaction (HCR) was chosen since it penetrates tissue efficiently (Choi et al., 2010), allows strong signal amplification, has high signal-to-noise with background-reducing probe design (Choi et al., 2018), and permits multiple rounds of stripping and re-probing (Nicovich et al., 2019; Fig.3, Extended Data Fig. 2. Following MPC recordings (Fig. 2b-g), slices were fixed, passively cleared, and stained by $\mathrm{mFISH}$ using HCR kit version 3.0 (Shah et al., 2016; Choi et al., 2018). Messenger RNA from excitatory (SLC17A7) and inhibitory (GADI) marker genes were easily resolved in both patched (biocytin/streptavidin, StAv) and neighboring nonpatched neurons (Extended Data Fig. 3a-d). As expected, SLC17A7 and GAD1 expression was mutually exclusive in excitatory and inhibitory neurons, respectively, and only $G A D 1^{+}$cells were found in layer 1 . Importantly, SLC17A7 mRNA staining was comparable between patched and neighboring non-patched neurons after a long whole-cell recording indicating that dialysis of mRNA from the cell during recording was not detectable in excitatory neurons (around 30-75 min; Extended Data Fig. 3b,c,i,j). We were also able to resolve SLC17A7 and GAD1 mRNA staining through the depth of the slice and didn't observe any significant changes of averaged fluorescent intensities in individual neurons (Extended Data Fig. 3j,k).

Studying connectivity between defined interneuron subclasses in acute human slice preparations is challenging because 1) GABAergic interneurons are difficult to identify during acute MPC experiments since they are not abundant ( 20-30\% of cortical neurons; Fig. 2j-1), and 2) unambiguous identification of interneuron subclass identity (e.g. PVALB, SST, VIP, LAMP5) has not been reliable with post-hoc immunohistochemistry in both non-human primate and human tissues. For example, PVALB antibodies works well (Szegedi et al., 2017), but SST and VIP antibodies do not work reliably in our hands (data not shown). Here, the GABAergic interneuron subclasses PVALB, SST, and VIP were readily resolved using HCR and RNA transcript probes for PVALB, SST, VIP and LAMP5 (lower panel of Extended Data Fig. 2). Inhibitory neurons were occasionally recorded during the acute MPC experiments. For example, a fast spiking interneuron (Reyes et al., 1998; cell4 in Fig. 2b) revealed strong excitatory postsynaptic potential (EPSP) responses that rapidly depress (e.g., cell1 to cell4 and cell2 to cell4), compared to small responses with weakly depressing characteristics in pyramidal neurons from presynaptic pyramidal neuron (e.g., cell3 to cell5; Fig. 2b,c). The intrinsic features (Fig. 2d-g) and morphology (Fig. 2h,i) of this cell were consistent with the identity of a PVALB cell type, although PVALB mRNA staining was very weak. This was seen with several patched $P V A L B^{+}$cells, where $P V A L B$ mRNA abundance was at lower levels than adjacent unpatched $P V A L B^{+}$cells (Extended Data Fig. 3e-g). Whether this reflects real differences in mRNA abundance between cells, or dilution or leakage of this subclass mRNA through patch pipette during whole-cell recording is unclear. Human tissue often exhibits dense lipofuscin around some somatic structures (Fig. 2j-1, Extended Data Fig. 3e-g), and persists after tissue clearing with 8\% SDS and throughout the mFISH staining procedure. However, it was possible to distinguish the distribution of amplified mRNA fluorescent dots from lipofuscin autofluorescence by imaging across multiple channels, as lipofuscin is fluorescent in all channels (e.g. Extended Data Fig. 3e-g).

\section{MPC electrophysiology in virally transduced human ex vivo cultured cortical slices}

To overcome the difficulty in targeting diverse interneuron subclasses, we performed rapid viral genetic labeling of cortical GABAergic interneurons. We applied an adeno-associated virus (AAV) vector that drives SYFP2 reporter express under the control of an optimized forebrain GABAergic neuron enhancer (Stuhmer et al., 2002; Dimidschstein et al., 2016) in human organotypic slice culture (see Methods; Ting et al., 2018; Mich et al., 2020). Inhibitory subclass identity was established post-hoc by mFISH staining. Virus was directly applied to the slice surface at a concentration of $1-5 \mathrm{e}^{10} \mathrm{vg} / \mathrm{slice}$. Fast reporter expression allowed us to execute physiology 
experiments between 2.5 and 7 days after viral administration. We performed targeted MPC recording with AAVDLX2.0-SYFP2 labeled cortical slices at 7 DIV (Fig. 3a; see also the example traces of connectivity assay performed in 2.5 DIV with viral labeling, Extended Data Fig. 1). SYFP2 ${ }^{+}$neurons were targeted to evaluate the connectivity between interneurons. Inhibitory postsynaptic potential (IPSP) responses were detected in multiple connected cell pairs (cell6 to cell8 and cell9 to cell8; Extended Data Fig. 4a). Spike trains with 8 pulses at 20 $\mathrm{Hz}$ in the presynaptic cell induced synaptic depression of IPSP responses (Fig. 3g,h). Recovery from synaptic depression was measured by probing with additional 4 pulses after variable inter-spike intervals $(250 \mathrm{~ms}, 500 \mathrm{~ms}$, $1 \mathrm{~s}, 2 \mathrm{~s}$ and $4 \mathrm{~s}$ ) following induction by the 8 pulses spike train at $50 \mathrm{~Hz}$ (Fig. $\mathbf{3 g}, \mathbf{h})$. Occasionally, electrical synapses mediated by gap junctions were detected between interneurons (Fig. 3c). Intrinsic membrane properties of patched interneurons were investigated with step depolarization (Fig. 3d-f, Extended Data Fig. 4b) and they appear indistinguishable from human cortical interneuron properties measured in acute slices (Fig. 3d-f $v s$ Fig. 2d-g).

Cell subclass identity was subsequently established using multi-round mFISH staining and mRNA signals were quantified (Fig. 3i-m, Extended Data Figs. 5\&6). We didn't observe any noticeable difference of mRNA staining signals between patched and neighboring non-patched neurons in slice culture (Extended Data Fig. 6a). The ability to stain across multiple rounds allowed probing for an increased number of genes, and re-probing for genes that produced low signal from the first round such as VIP (Fig. 3k-m cell3). Axonal and dendritic morphologies were then reconstructed based on biocytin fills and streptavidin staining (Fig. 3j) as was done in acute slices (Fig. 2h,i), to allow comparison of morphologies across preparations. All ten patched cells were GABAergic, and multiple VIP, SST, and PVALB-positive cells were patched from two sets of MPC recording attempts (box i,ii of Fig. 3i). This experiment shows how application of a cell class specific AAV vector facilitates highly efficient prospective cell class labeling and subsequent identification of cortical interneuron subclasses that are difficult to reliably target in acute brain slice preparations.

We noted some differences between MPC recordings in viral labeled slice culture and acute slice preparation. First, giga-ohm seals were more readily obtained between patch pipette and cell membrane in neurons from ex vivo cultured slices compared to acute slices. Second, although patching reporter-labeled cells was easier, the somatic structure of unlabeled neurons was more difficult to resolve in slice culture with minimal positive pressure on the patch pipette, making patching unlabeled neurons more challenging. Nonetheless, the ability to exclusively target genetically labeled GABAergic neuron subclasses in the human neocortex greatly improved throughput and efficiency of targeted recording experiments. In summary, we demonstrate techniques that allow efficient analysis of synaptic connectivity and intrinsic membrane properties in specific neuron subclasses in human brain tissues, both in acute slices (Fig. 2) and slice cultures using viral reporters for prospective labeling (Fig. 3).

\section{DISCUSSION}

Major advances in understanding the cellular makeup of complex brain tissues are being driven by single cell transcriptomic technologies combined with large-scale brain atlasing efforts to create molecularly defined cell taxonomies in mouse, monkey and human. In mice, molecular characterization of cell types (Zeisel et al., 2015; Tasic et al., 2018; Ximerakis et al., 2019) has led to increasingly refined tools for genetic access and highly granular characterization of neuronal circuit elements (He et al., 2016; Daigle et al., 2018). In contrast, understanding fine-grained microcircuitry in human tissue as well as in non-genetically tractable organisms has lagged far behind. A major challenge therefore is to develop techniques that allow a similar analysis of molecularly defined circuit elements for biomedical research. Significant advances in in vitro slice physiology and slice culture on human neurosurgical specimens, as well as in the development of AAV vectors for rapid infection and cell type-specific transgene expression, provide new avenues for targeted analysis of specific cell 
types in the brain. These methods provide a means to study neuronal and circuit properties in human neocortex and link them to emerging molecular classifications of cell types with a combination of prospective labeling and post-hoc identification with marker gene panels.

As demonstrated here, mFISH with multiple rounds of staining can be executed on cleared thick in vitro human slice preparations, preserving tissue integrity and cell morphology, thereby allowing molecular identification of synaptically connected neurons using robust marker genes for neuron subclasses. We showed that this methodological approach of quadruple modality functional characterization can be extended to slice culture with viral labeling as well. This approach is well poised to take advantage of an increasing number of enhancer-driven subclass and type-specific viral tools (Dimidschstein et al., 2016; Hrvatin et al., 2019; Jüttner et al., 2019; Mehta et al., 2019; Nair et al., 2019; Mich et al., 2020; Graybuck et al., 2020; Vormstein-Schneider et al., 2020) to allow prospective targeting of cell classes or subclasses, and subsequent refinement of cell type identity by a combination of marker genes using mFISH. Brain slice culturing and viral transgenesis will allow not only measurement, but also functional manipulation of the human microcircuit, e.g. via optogenetic approaches. Together, these methods provide unprecedented ability to probe the fine circuit architecture of the human neocortex and can accelerate progress towards uncovering conserved and specialized features of human brain circuit organization and function.

A number of challenges were identified for future improvement. Although mRNA labeling was robust for abundant genes, less abundant genes were more difficult to detect. Autofluorescence from lipofuscin, a common feature of human brain tissue, can complicate analysis and obscure mRNA signal. Improvement of lipofuscin mitigation techniques will facilitate future analysis. In some cases, we did not readily detect expected mRNA transcripts for cells with certain types of electrophysiological features (such as fast-spiking inhibitory neurons that would be expected to express PVALB). This could be the true state of the cell, or due to loss of mRNA through the patch pipette or leakage from the cell after pipette withdrawal. Finally, greater cell type resolution will be gained through the use of more highly multiplexed mFISH techniques (Chen et al., 2015; Eng et al., 2019). Despite these opportunities for further refinement, the approach outlined here enables unprecedented quadruple modality functional interrogation of human brain cell types and is a valuable step toward deciphering the correspondence between these multiple data modalities.

\section{Online content}

Any methods, additional references, Nature Research reporting summaries, source data, extended data, supplementary information, acknowledgements, peer review information; details of author contributions and competing interests; and statements of data and code availability are available at http://

\section{Data availability}

The electrophysiology and imaging data sets that support the findings of this study are available from the corresponding authors upon reasonable request.

\section{Code availability}

The software codes used for the analyses are available from the corresponding authors upon reasonable request. 


\section{References}

Andersson, M. et al. Optogenetic control of human neurons in organotypic brain cultures. Sci Rep 6, 24818 doi:10.1038/srep24818 (2016).

Beaulieu-Laroche, L. et al. Enhanced dendritic compartmentalization in human cortical neurons. Cell 175, 643651 (2018).

Berg, J. et al. Human cortical expansion involves diversification and specialization of supragranular intratelencephalic-projecting neurons. bioRxiv preprint doi: 10.1101/2020.03.31.018820 (2020).

Chen, K.H. et al. RNA imaging. Spatially resolved, highly multiplexed RNA profiling in single cells. Science 348, aaa6090 (2015).

Choi, H.M.T. et al. Programmable in situ amplification for multiplexed imaging of mRNA expression. Nat. Biotechnol. 28, 1208-1212 (2010).

Choi, H.M.T. et al. Third-generation in situ hybridization chain reaction: multiplexed, quantitative, sensitive, versatile, robust. Development 145, doi: 10.1242/dev.165753 (2018).

Daigle, T.L. et al. A suite of transgenic driver and reporter mouse lines with enhanced brain-cell-type targeting and functionality. Cell 174, 465-480.e22 (2018).

Dimidschstein, J. et al. A viral strategy for targeting and manipulating interneurons across vertebrate species. Nat Neurosci 19, 1743-1749 (2016).

Eng, C.H.L. et al. Transcriptomic-scale super-resolved imaging in tissues by RNA seqFISH. Nature 568, 235239 (2019).

Eugene, E. et al. An organotypic brain slice preparation from adult patients with temporal lobe epilepsy. $J$. Neurosci. Methods 235, 234-244 (2014).

Graybuck, L.T. et al. Enhancer viruses and transgenic platform for combinatorial cell subclass-specific labeling. bioRxiv preprint doi: 10.1101/ 525014 (2020).

He, M. et al. Strategies and tools for combinatorial targeting of GABAergic neurons in mouse cerebral cortex. Neuron 91, 1228-1243 (2016).

Hrvatin, S. et al. A scalable platform for the development of cell-type-specific viral drivers. Elife 8, pii: e48089, doi:10.7554/eLife.48089 (2019).

Hodge, R.D. et al. Conserved cell types with divergent features in human versus mouse cortex. Nature 573, 6168 (2019).

Jiang, M. et al. Enhancement of asynchronous release from fast-spiking interneuron in human and rat epileptic neocortex. PLoS Biol. 10, e1001324. doi: 10.1371/journal.pbio.1001324. (2012).

Jüttner, J. et al. Targeting neuronal and glial cell types with synthetic promoter AAVs in mice, non-human primates and humans. Nat Neurosci. 22, 1345-1356 (2019).

Kalmbach, B.E. et al. h-channels contribute to divergent intrinsic membrane properties of supragranular pyramidal neurons in human versus mouse cerebral cortex. Neuron 100, 1194-1208 (2018).

Lake, B.B. et al. Neuronal subtypes and diversity revealed by single-nucleus RNA sequencing of the human brain. Science 352, 1586-1590 (2016).

Le Duigou, C. et al. Imaging pathological activities of human brain tissue in organotypic culture. J. Neurosci. Methods 298, 33-44 (2018).

Mehta, P. et al. Functional access to neuron subclasses in rodent and primate forebrain. Cell Rep 26, 2818-2832 (2019).

Mich, J.K. et al. Functional enhancer elements drive subclass-selective expression from mouse to primate neocortex. bioRxiv preprint doi: 10.1101/555318 (2020).

Molnar, G. et al. Complex events initiated by individual spikes in the human cerebral cortex. PLoS Biol. 6, e222 doi: 10.1371/journal.pbio.0060222 (2008). 
Nair, R.R. et al. Generation of viral vectors specific to neuronal subtypes of targeted brain regions by EnhancerDriven Gene Expression (EDGE). bioRxiv preprint doi: 10.1101/606467 (2019).

Nicovich, P.R. et al. Multimodal cell type correspondence by intersectional mFISH in intact tissues. bioRxiv preprint doi: 10.1101/525451 (2019).

Peng, Y. et al. High-throughput microcircuit analysis of individual human brains through next-generation multineuron patch-clamp. Elife 8 pii: e48178, doi: 10.7554/eLife.48178 (2019).

Pfeffer, C.K. et al. Inhibition of inhibition in visual cortex: the logic of connections between molecularly distinct interneurons. Nat Neurosci. 16, 1068-1076 (2013).

Reyes, A. et al. Target-cell-specific facilitation and depression in neocortical circuits. Nat Neurosci. 1, 279-285 (1998).

Schwarz, N. et al. Human cerebrospinal fluid promotes long-term neuronal viability and network function in human neocortical organotypic brain slice cultures. Sci Rep 7, 12249, doi:10.1038/s41598-017-12527-9 (2017). Schwarz, N. et al. Long-term adult human brain slice cultures as a model system to study human CNS circuitry and disease. Elife 8 pii: e48417, doi: 10.7554/eLife.48417 (2019).

Seeman, S.C. et al. Sparse recurrent excitatory connectivity in the microcircuit of the adult mouse and human cortex. Elife 7 pii: e37349, doi: 10.7554/eLife.37349 (2018).

Shah, S. et al. Single-molecule RNA detection at depth by hybridization chain reaction and tissue hydrogel embedding and clearing. Development 143, 2862-2867 (2016).

Stuhmer, T. et al. Expression from a Dlx gene enhancer marks adult mouse cortical GABAergic neurons. Cereb. Cortex 12, 75-85 (2002).

Szabadics, J. et al. Excitatory effect of GABAergic axo-axonic cells in cortical microcircuits. Science 311, 233235 (2006).

Szegedi, V. et al. High-precision fast-spiking basket cell discharges during complex events in the human neocortex. eNeuro 4 ENEURO.0260-17.2017. doi: 10.1523/ENEURO.0260-17 (2017).

Tasic, B. et al. Shared and distinct transcriptomic cell types across neocortical areas. Nature 563, $72-78$ (2018).

Testa-Silva, G. et al. High bandwidth synaptic communication and frequency tracking in human neocortex. PLoS Biol. 12, e1002007 doi: 10.1371/journal.pbio.1002007 (2014).

Tian, C. et al. Molecular identity of axonal sodium channels in human cortical pyramidal cells. Front Cell Neurosci 8, 297 doi: 10.3389/fncel.2014.00297 (2014).

Ting, J.T. et al. A robust ex vivo experimental platform for molecular-genetic dissection of adult human neocortical cell types and circuits. Sci Rep 8, 8407, doi:10.1038/s41598-018-26803-9 (2018).

Tremblay, R. et al. GABAergic interneurons in the neocortex: from cellular properties to circuits. Neuron 91, 260-292 (2016).

Verhoog, M.B. et al. Mechanisms underlying the rules for associative plasticity at adult human neocortical synapses. J. Neurosci. 33, 17197-17208 (2013).

Vormstein-Schneider, D. et al. Viral manipulation of functionally distinct interneurons in mice, non-human primates and humans. Nat Neurosci. doi: 10.1038/s41593-020-0692-9 (2020).

Ximerakis, M. et al. Single-cell transcriptomic profiling of the aging mouse brain. Nat Neurosci. 22, 1696-1708 (2019).

Wang, B. et al. A subtype of inhibitory interneuron with intrinsic persistent activity in human and monkey neocortex. Cell Rep 10, 1450-1458 (2015).

Zeisel, A. et al. Brain structure. Cell types in the mouse cortex and hippocampus revealed by single-cell RNAseq. Science 347, 1138-1142 (2015). 


\section{a Tissue processing}

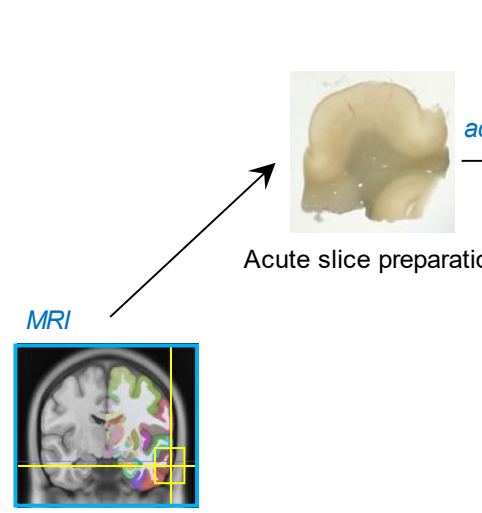

Resected human cortical slice tissue
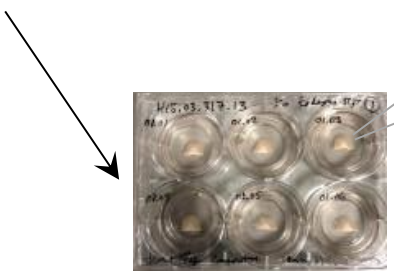

Slice culture preparation b in vitro electrophysiology

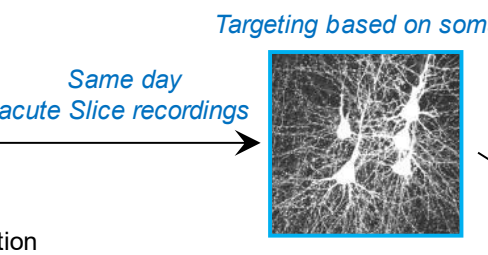

Multi-patch-clamp recordings in acute slice
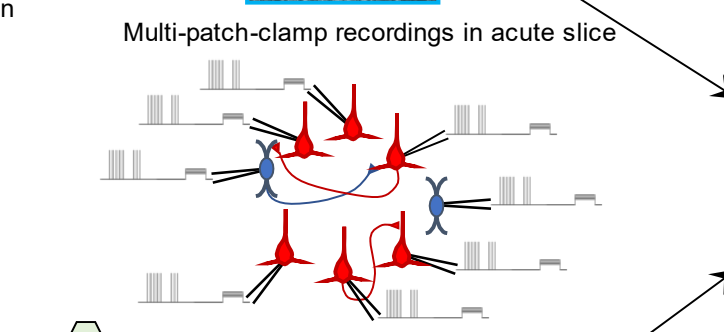

Targeting fluorescently labeled neurons

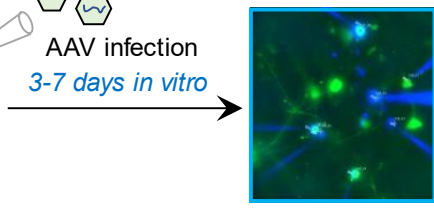

Multi-patch-clamp recordings in slice culture
C Post-hoc identification of gene expression $\&$ morphological reconstruction

Fig. 1 | Schematic of experimental workflow. a, Human neocortical tissue from neurosurgical resection enters either acute slice preparation within 45 min following scalpel excision from the patient (upper) or organotypic slice culture preparation with viral transduction (lower). b, Up to eight simultaneous patch-clamp recordings are performed on either acute slices (upper) or slice culture after 2.5 to 7 days in vitro (lower). Targeting of neurons is either carried out by visually identifying cell bodies using an upright microscope with oblique illumination (upper) or by targeting neurons expressing fluorescent reporter following viral infection (lower). c, Multiplexed fluorescence in situ hybridization (mFISH) and biocytin/streptavidin staining for morphological reconstruction is performed on fixed slices. 


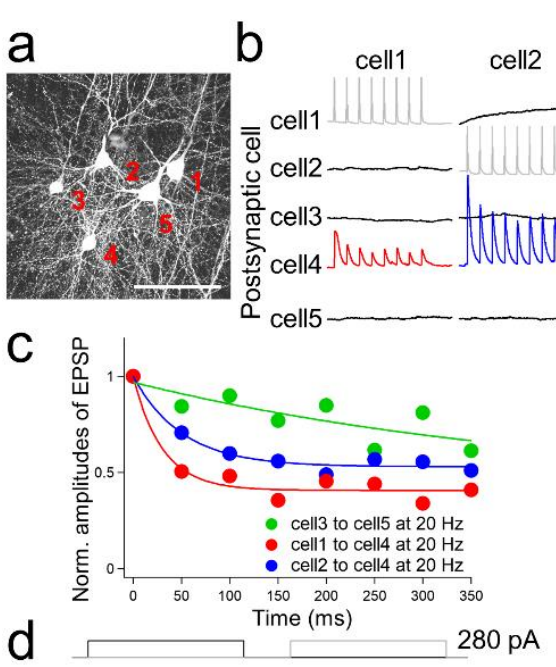

$280 \mathrm{pA}$
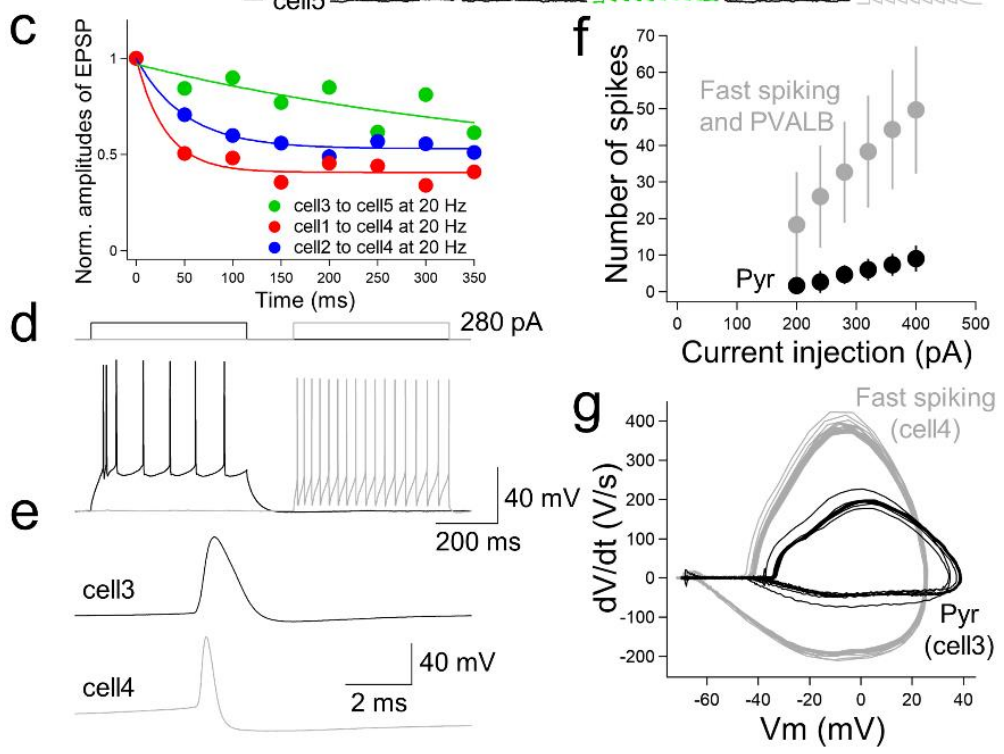
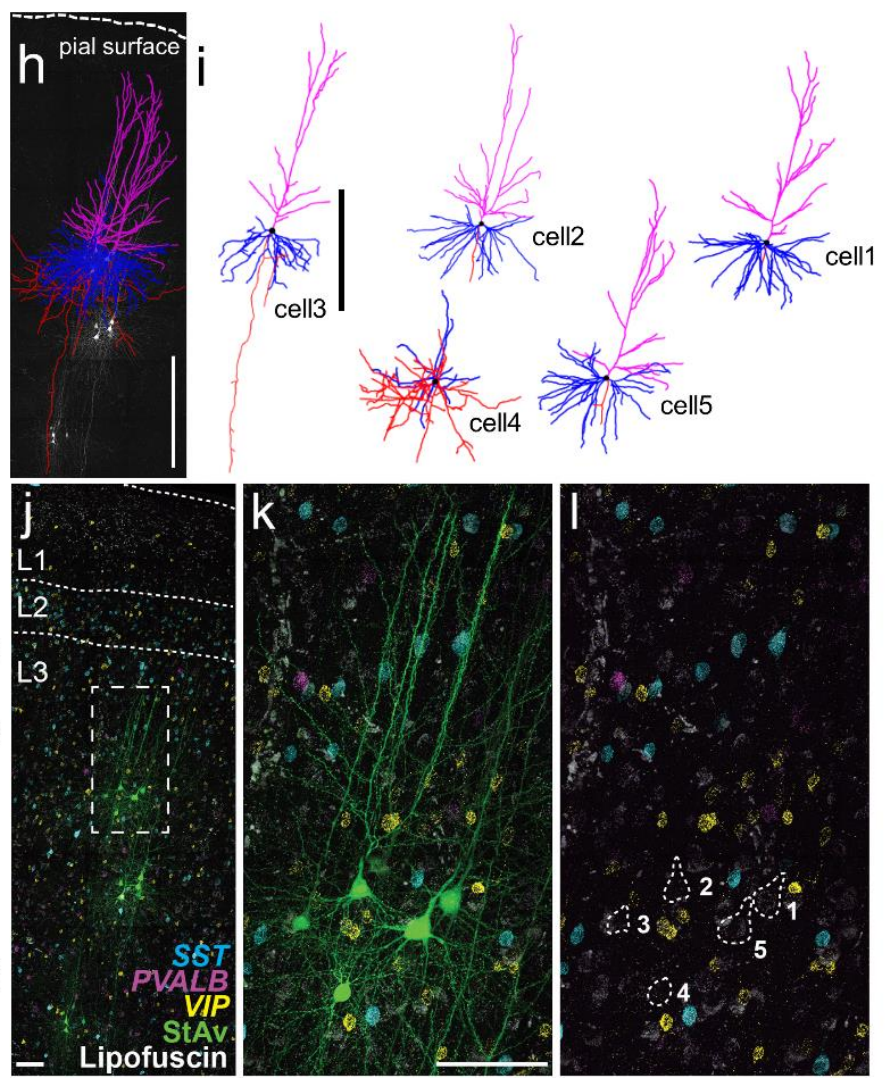

Fig. 2 | Quadruple modality data in acute ex vivo human neocortex. An example of an experiment using the acute slice preparation with five cells simultaneously patched. a, Maximum intensity projection montage confocal image of biocytin/streptavidin labeling. Scale bar, $50 \mu \mathrm{m}$. b, Corresponding membrane voltage traces of connectivity assay. Presynaptic action potentials (gray) in individual neurons (cell1 to cell5) were sequentially generated by 8 brief current pulses at $20 \mathrm{~Hz}$ while simultaneously recording the postsynaptic membrane voltage in non-stimulated neurons in current-clamp mode (black). Traces were averaged with 10 repetitive 8 pulse stimulation. This probing uncovers a strong and adapting excitatory synaptic connection from cell 2 to cell 4 (blue trace) and cell1 to cell4 (red trace) compared to the synaptic connection from cell3 to cell5 (green). c, Summary plot of short-term synaptic dynamics with presynaptic $20 \mathrm{~Hz}$ stimulation (8 pulses at $20 \mathrm{~Hz}$ ) in connected pairs shown in b. Amplitude is normalized to the size of the initial EPSP. d, Example traces of action potential generation by step current injection in regular spiking (cell3, black) and fast spiking neuron (cell4, gray). The same amount of current injection (280 pA) was applied to cell3 and cell4. e, Spike shape comparison between regular and fast spiking neurons detected in the connectivity assay shown in $\mathbf{b}$. $\mathbf{f}$, a frequency-current curve of pyramidal neuron (Pyr; mean \pm standard deviation, $n=3$ ), and fast spiking neuron (panel $\mathbf{k}$, cell4) and PVALB positive neurons (upper 2 cells marked as dotted lines, panel g of Extended Data Fig. 3) (Fast spiking and PVALB; mean \pm standard deviation, $n=3)$. g, Phase plot (dV/dt vs V) analysis based on the responses shown in d. h, Morphological reconstruction of the 5 recorded neurons shown in a. Scale bar, $500 \mu \mathrm{m}$. i, Reconstruction of individual neurons. Scale bar, $500 \mu \mathrm{m}$. Blue, magenta, and red indicate basal dendrites, apical dendrites, and axons in pyramidal neurons (cell 1,2,3,5). For the interneuron (cell4), blue and red indicate dendritic and axonal structures, respectively. j, Fluorescence montage of cells imaged in $\mathbf{a}, \mathbf{j}$-l stained by mFISH for inhibitory neuron subclass markers (PVALB, SST, and VIP) and biocytin. MPC recordings were performed on three separate cell clusters in this slice (j). Note, substantial lipofuscin is observed in this slice. White box in $\mathbf{j}$ is shown at higher magnification for mFISH and biocytin (k), or mFISH only (l). 

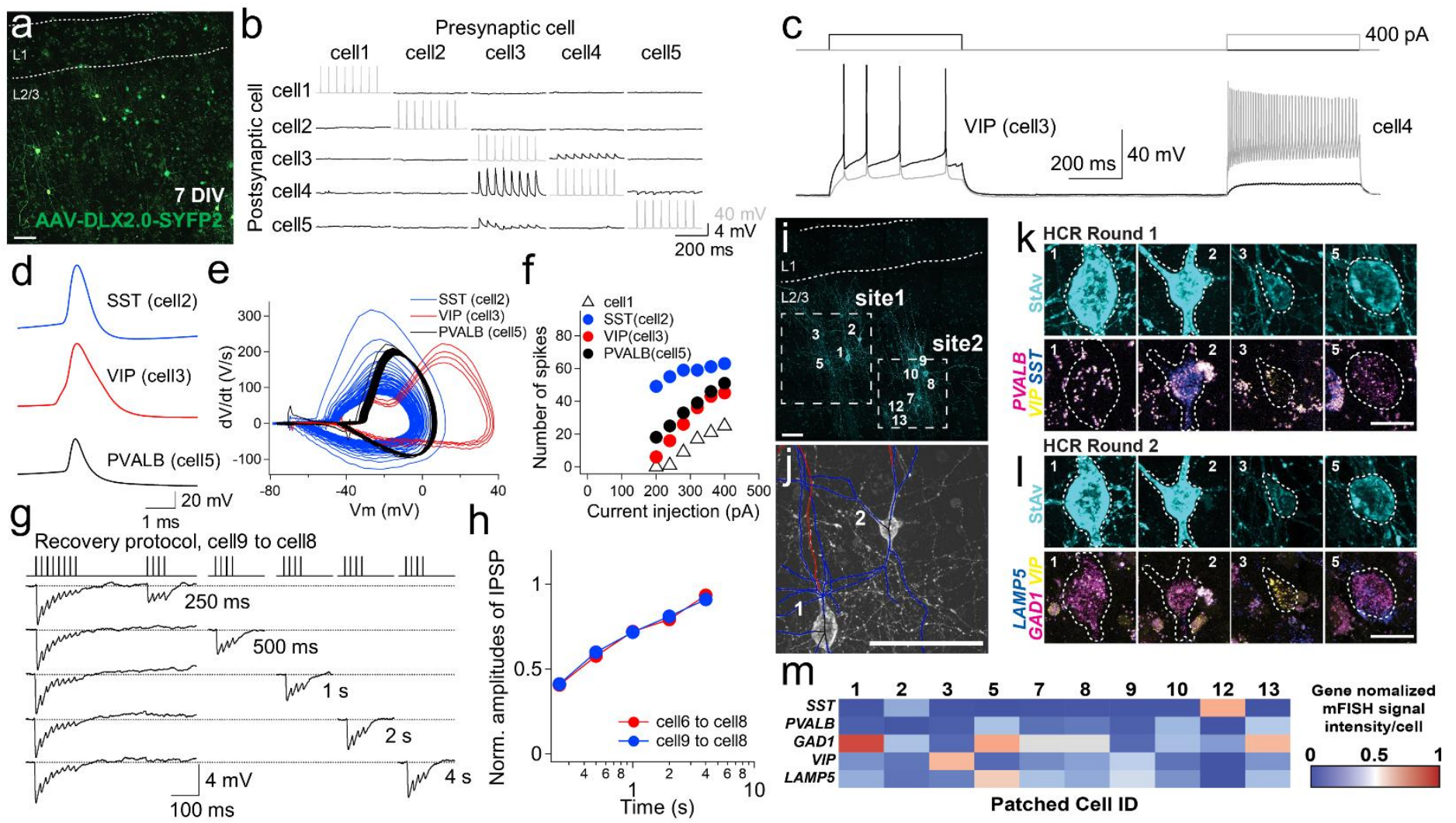

Fig. 3 | Quadruple modality data from AAV-labeled cortical GABAergic interneurons. a, Fluorescence montage of SYFP2 expression from a GABAergic neuron enhancer AAV (AAV-DLX2.0-SYFP2) in ex vivo human neocortex after seven days in culture ( 7 DIV refer to 7 days in vitro). b, Traces of local synaptic connectivity of patched neurons (shown in site 1 of panel $\mathbf{i}$; note that cell numbers in $\mathbf{i}$ are corresponding to the cell numbers in panels $\mathbf{b}-\mathbf{h}$, and $\mathbf{j}-\mathbf{m}$ ). Traces were averaged over 5 repetition of the 8 pulses stimulation at $20 \mathrm{~Hz}$. c, Example of gap junctional connectivity between interneurons. Note that current injection from one cell propagated to another cell and synchronized timing of action potentials. Cell3 (left; black trace) was identified as $V I P^{+}$by HCR staining but we failed to detect the subclass level identity of cell4 (right; gray trace) see panel $\mathbf{l}$, m). d, Spike shape examples detected in different subclass interneurons, i.e., SST (cell2), VIP (cell3), and PVALB (cell5) by HCR staining shown in panels k-m. e, Phase plot (dV/dt vs V) analysis based on the step current injection of $400 \mathrm{pA}$ in 3 subclass cell types detected in this experiment (panel e). f, Frequency-current curves from the trial shown in site1 of panel i. g, For the connectivity assay, an additional 4 presynaptic pulses were applied a variable interval $(250 \mathrm{~ms}, 500 \mathrm{~ms}, 1 \mathrm{~s}, 2 \mathrm{~s}$, and $4 \mathrm{~s})$ after the 8 pulses stimulation protocol to assay recovery from depression. Connected pairs from cell9 to cell8, averaged 5 times). $\mathbf{h}$, Time course of recovery from depression. Two examples are displayed with connected pairs (cell6 to cell8, cell9 to cell8). i, Fluorescent montage images of two neuron clusters evaluated by MPC recordings highlighted with boxes site1 and site 2 with patched cell numbers. Patched neurons displayed with biocytin/streptavidin staining (i) compared to SYFP2 fluorescence shown in panel a. Scale bar, $100 \mu \mathrm{m}$. j, Morphological reconstruction example. Blue indicates dendritic and red indicates axonal tracing. Scale bar, $50 \mu \mathrm{m}$. k-l, Some examples of HCR staining detection. High resolution images of individual patched cells stained by mFISH in round 1 (k) against for PVALB, SST, and VIP, and round 2 (l) for $L A M P 5, G A D 1$, and VIP. Scale bar, $10 \mu \mathrm{m}$. m, Expression level of each gene for each patched cell was quantified based on average intensity per cell. The average intensity was normalized by maximum value detected among the manually segmented patched and non-patched cells shown in Extended Data Fig. 6a. 

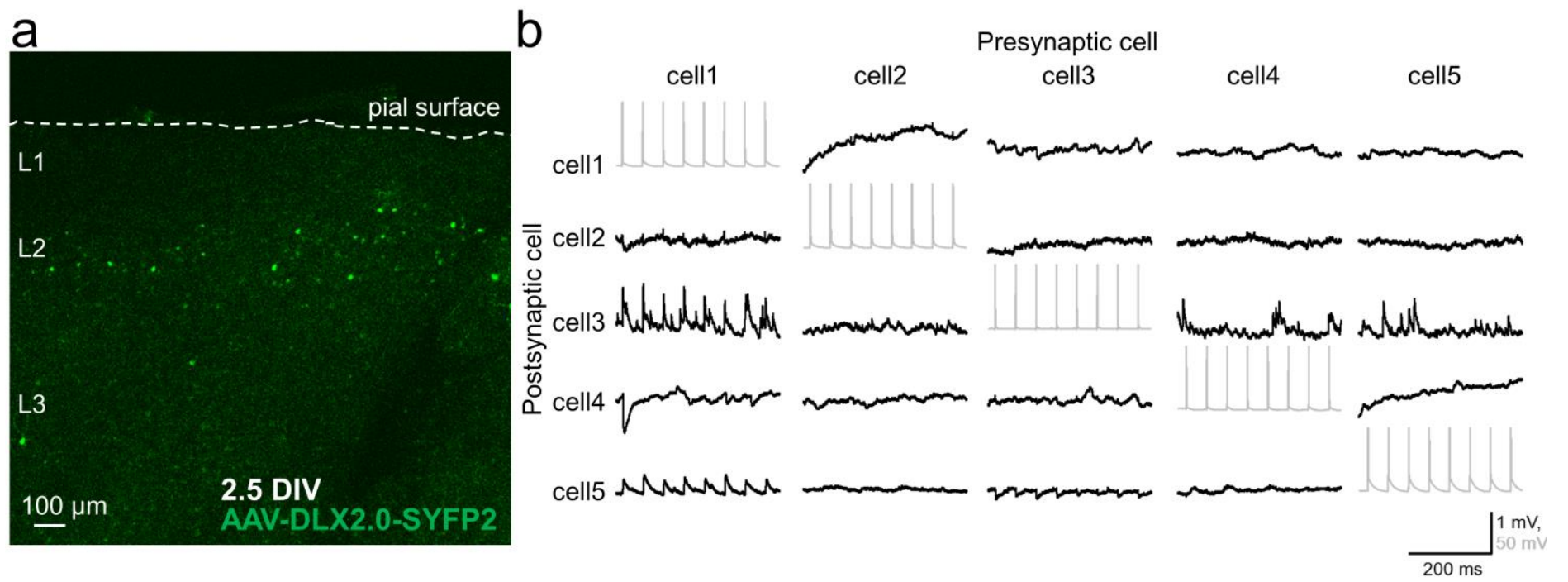

Normalized values are displayed with heat map (see also non-normalized values and heat map in Extended Data Fig. 6b). Note that some cells were successfully recorded with MPC electrophysiology but were not recovered after staining.

Extended Data Fig. 1 | Example of rapid expression of AAV-DLX2.0-SYFP2 virus labeling and corresponding MPC recording. Representative experiment was performed on 2.5 days in vitro (DIV) after AAV-DLX2.0-SYFP2 virus application. Fluorescently labeled neurons were routinely generated from this viral vector for MPC recordings at this early time point. a, Native SYFP2 viral labeling is shown in the area the electrophysiology experiment was performed. b, Connectivity assay with MPC recording. Traces were averaged with 10 repetitive stimuli. 


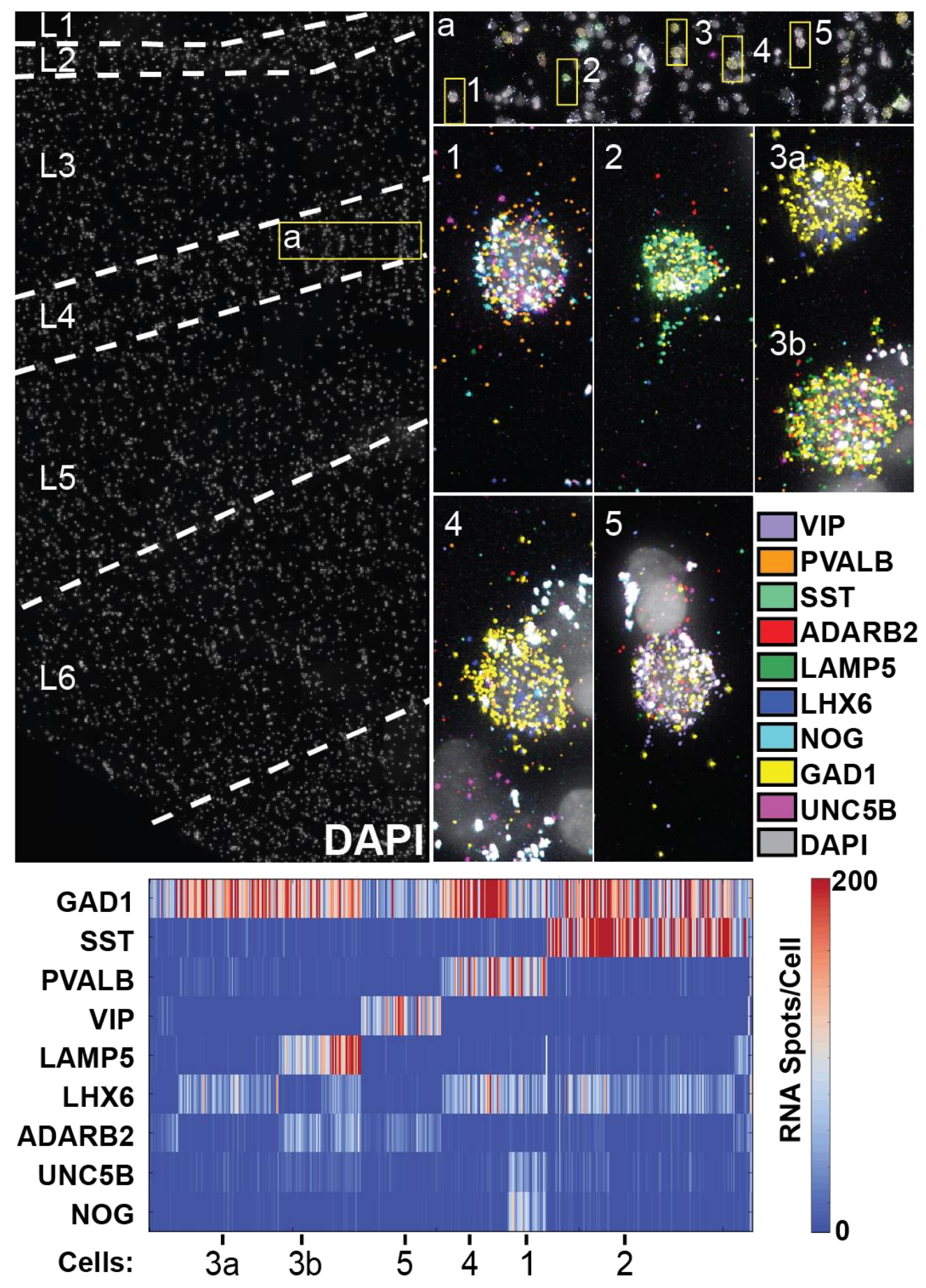

Extended Data Fig. 2 | GABAergic cell identification with multi-round HCR staining in thin frozen tissue. Nine GABAergic neuron marker genes were evaluated in frozen then thawed thin $(10 \mu \mathrm{m})$ slice preparation. Fluorescence montage with nuclei stained by DAPI (top left). Neocortical layers were determined from the DAPI stain. a, yellow boxed region (left) shown at higher resolution (right). The nine genes resolved the main cortical interneuron subclasses and one cell type (top right). RNA spots per cell are quantified (bottom), and largely reflect GABAergic subclasses observed in human transcriptomics studies (Lake et al., 2016; Hodge et al., 2019). The some of the same marker genes were used for MPC recording and post-hoc HCR staining in thick tissue preparation shown in Figs. 2,3, and Extended Data Figs. 3,5,6. 

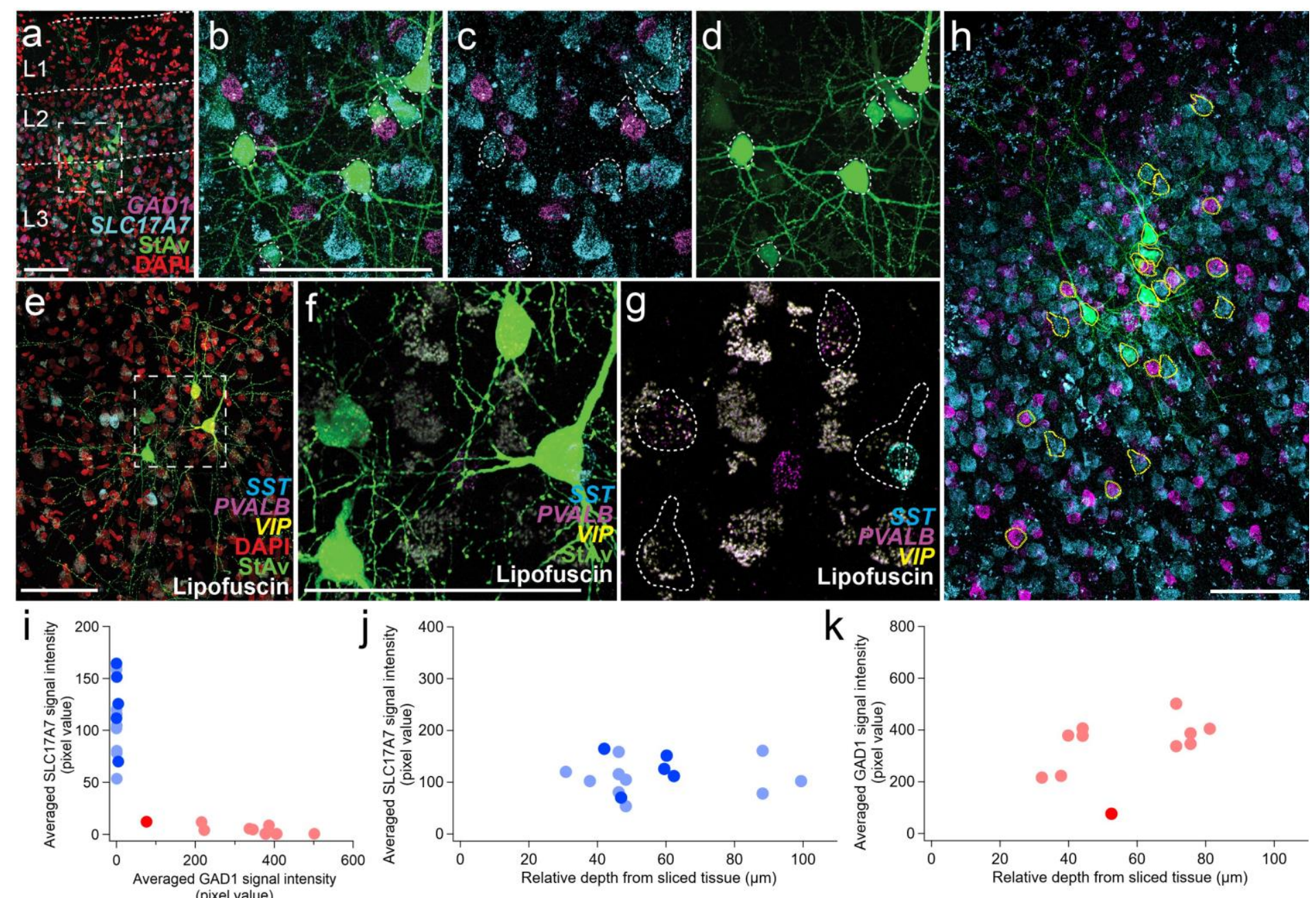

Extended Data Fig. 3 | Two examples of MPC experiments with excitatory and inhibitory HCR markers in acute ex vivo human neocortex and depth dependence of HCR signals. There are two examples of MPC experiments. One shown in a-d,h-k and the other shown in e-g. a, Fluorescence montage of maximum intensity projections showing mFISH staining for excitatory (SLC17A7) and inhibitory (GADl) neuron marker transcripts, nuclei (DAPI), and patched neurons (biocytin). Note that there is no SLC17A7 labeling in layer 1. (b-d) Enlargement of boxed region from a. e, Fluorescence montage showing four adjacent multi-patched cells from a different experiment. f-g, Four cells are shown, two of which are marked by the PVALB transcript. Note, although the cell on the right overlaps with $S S T$, this is a different Z-plane than the patched cells. The VIP transcript stained neurons are not shown in the field of view but some patched neurons showed VIP labeling in another region of this slice (data not shown). SLC17A7 and GAD1 mRNA staining signals were manually segmented and compared among the cells located in different depth from the slice surface (h-k). h, Yellow lines show 3D manually segmented neurons in a maximum intensity projection montage image that are used in panel i-k. i, SLC17A7 and $G A D 1$ expression is mutually exclusive. Blue indicates $S L C 17 A 7^{+}$neurons (patched neurons, blue; unpatched neurons, light blue) and red indicates $G A D 1^{+}$neurons (patched neurons, red; non-patched neurons, light red). Same cells are displayed with depth dependent manner $(\mathbf{j}-\mathbf{k})$. j, SLC17A7 ${ }^{+}$positive neurons are displayed along relative depth of the slice. $\mathbf{k}$, Similarly, $G A D 1^{+}$positive neurons are displayed along relative depth of the slice. Scale bars, $100 \mu \mathrm{m}$ in $\mathbf{a}, \mathbf{b , e , f , h}$. 
a

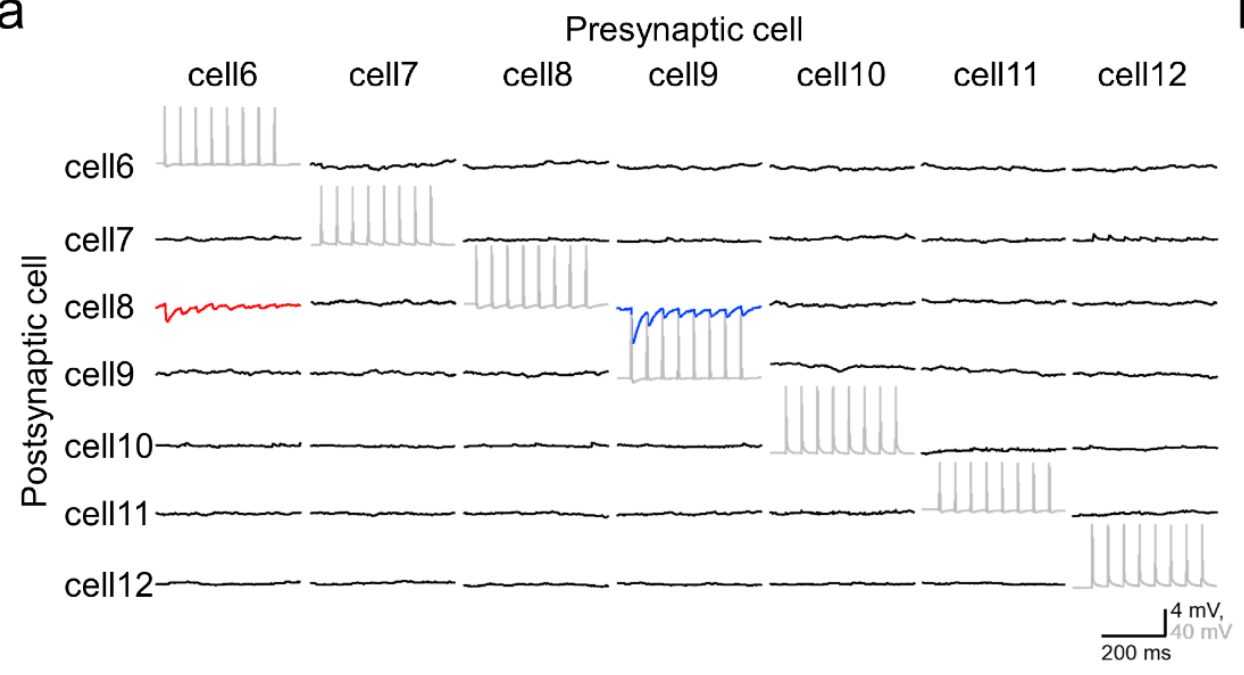

b

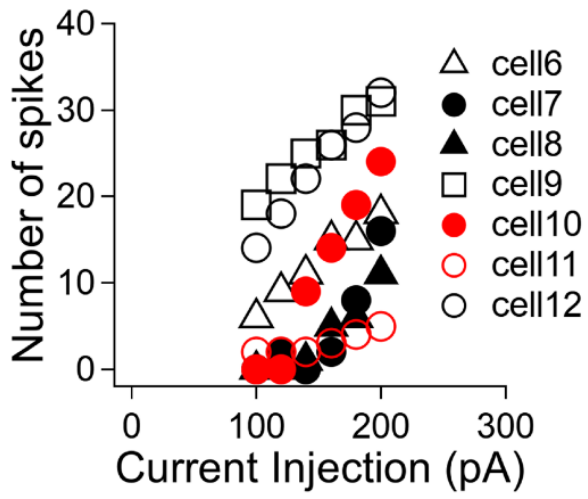

Extended Data Fig. 4 | Further information of local connectivity and frequency-current relationship from AAV-labeled cortical slice shown in Fig. 3. i (site2). Traces of local synaptic connectivity of patched neurons shown in site 2 . Traces were averaged over 5 repetition of the 8 pulses stimulation at $20 \mathrm{~Hz}$. Inhibitory connections from cell6 to cell8 (postsynaptic response; red) and from cell9 to cell8 (postsynaptic response; blue) are highlighted and recovery dynamics on these connections are shown in Fig. 3.g-h. b. Frequency-current curves from the trial shown in site $\mathbf{2}$ of panel $\mathbf{i}(\mathbf{F i g} . \mathbf{3})$. 

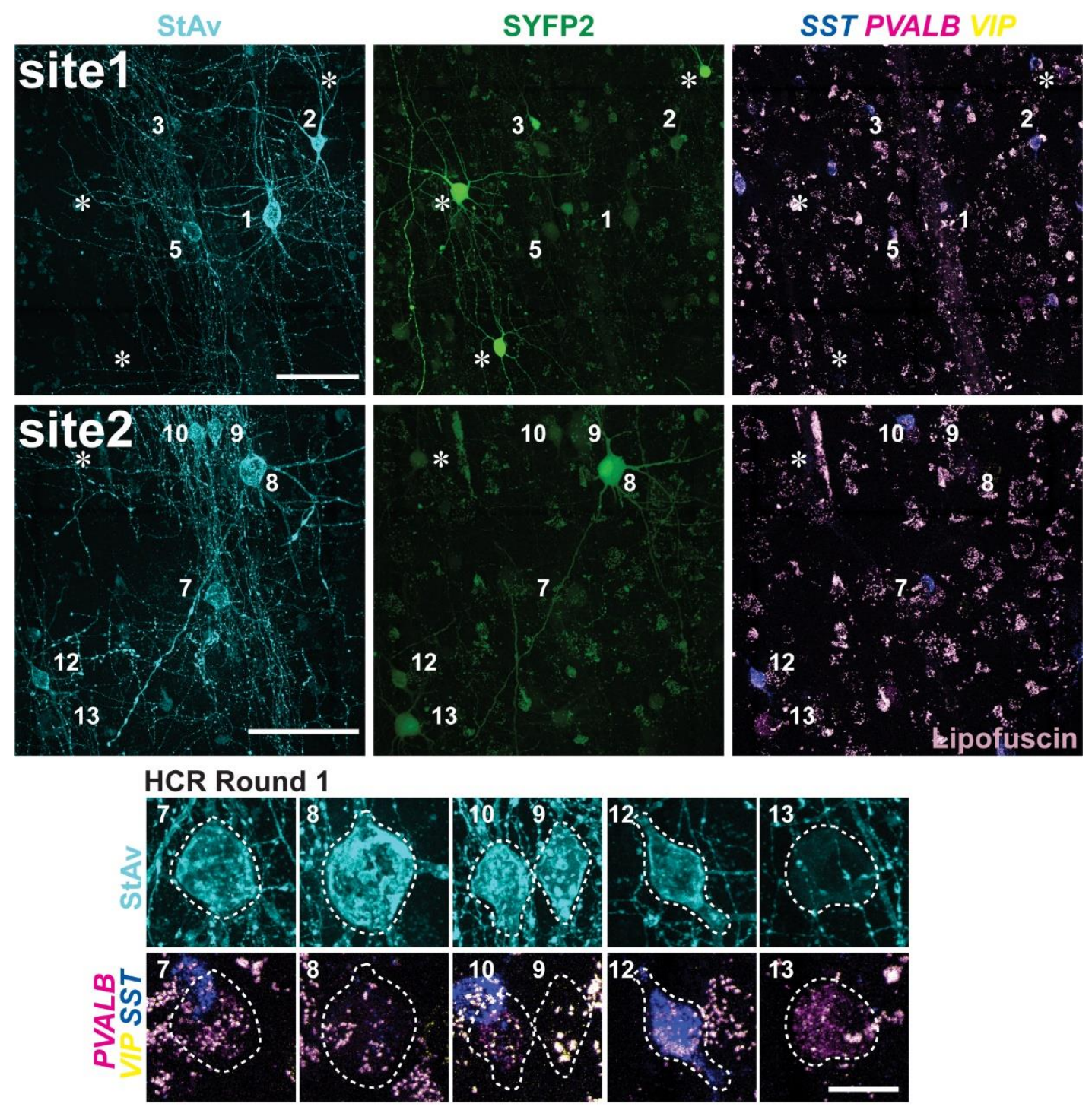

\section{HCR Round 2}
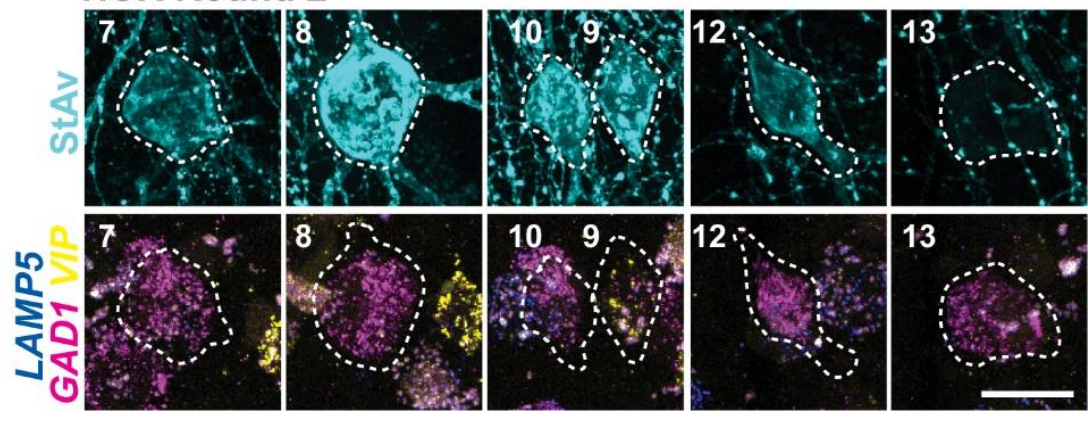

Extended Data Fig. 5 | Further information of HCR staining assays shown in Fig. 3. Upper panel, high magnification images of site1 and site2 shown in Fig. 3. Patched neurons displayed with biocytin/streptavidin only staining (StAv; left) or SYFP2 (SYFP2; middle), mFISH, and lipofuscin (light purple; right). Asterisks mark $\mathrm{SYFP}^{+}$cells not marked by biocytin/streptavidin. Scale bar, $100 \mu \mathrm{m}$. Lower panel, High resolution images of individual patched cells stained by mFISH in round 1 against PVALB, SST, and VIP, and round 2 against LAMP5, $G A D 1$, and VIP. Scale bar, $10 \mu \mathrm{m}$. Cell numbers are labeled consistently in Figure 3. 


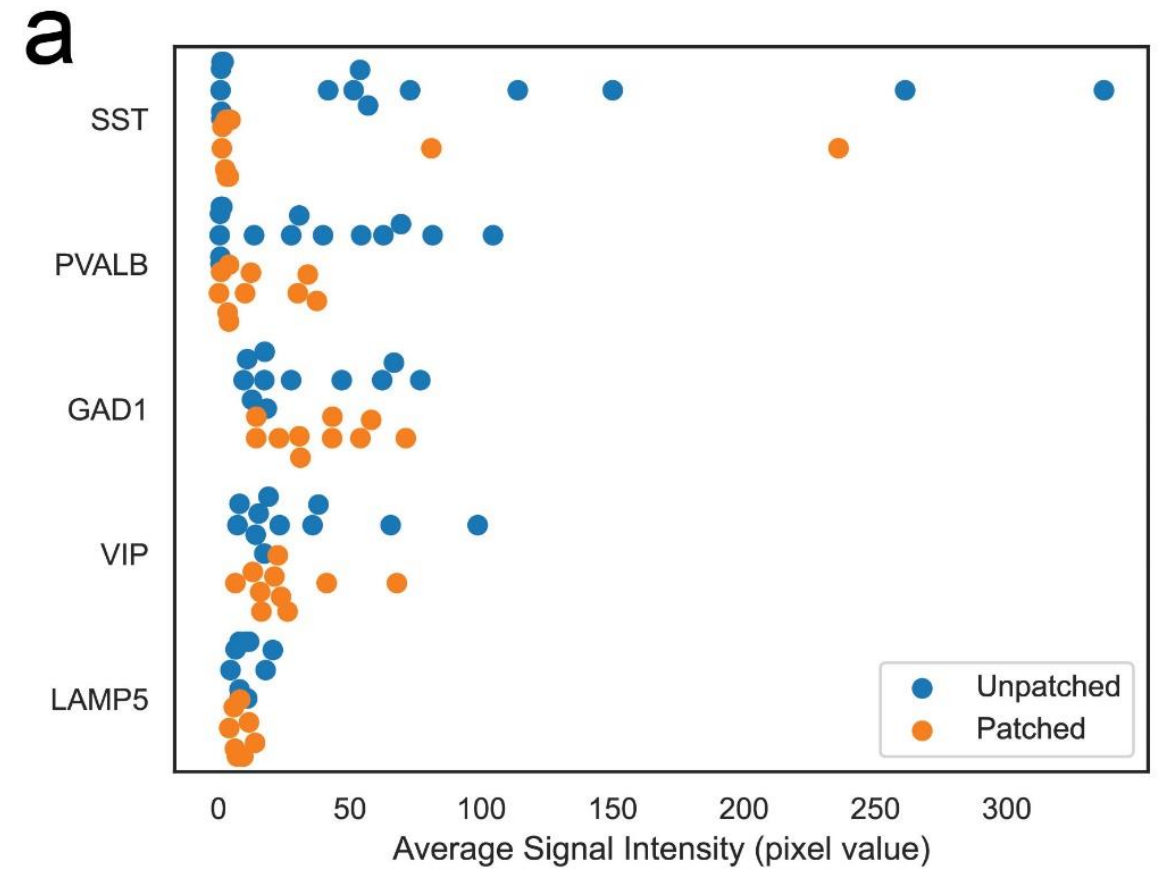

b

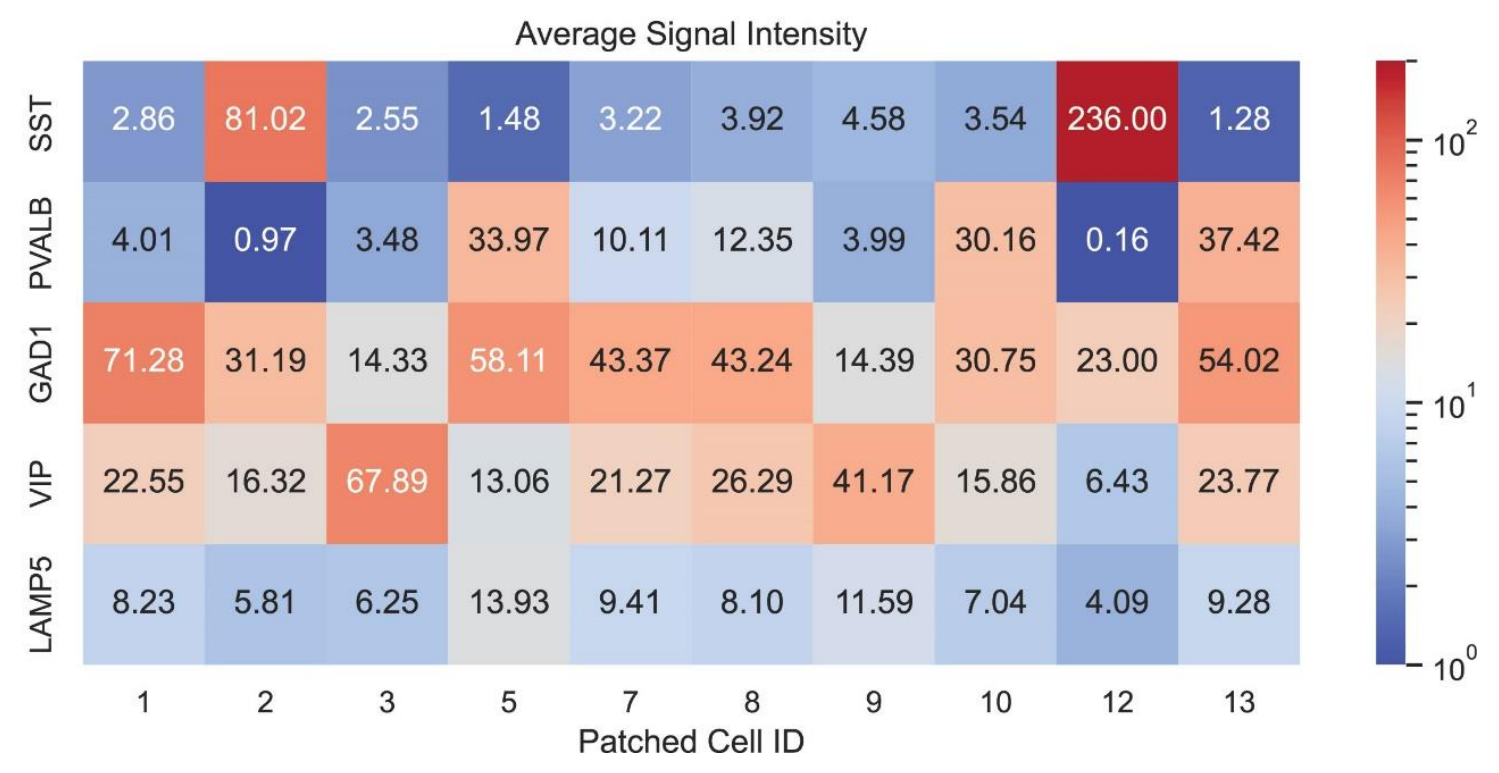

Extended Data Fig. 6 | Quantification of HCR signal in slice culture preparation (related to Figure 3 and Extended Data Figure 5). a, HCR signal comparison between patched and non-patched neighboring neurons. Both patched and non-patched neighboring neurons were manually segmented in 3D. Average intensity values of HCR signals in each neuron were quantified and displayed. Orange closed circles indicate the patched neurons shown in Figure 3 and blue closed circles indicate non-patched neighboring neurons. b, Heat map displaying average fluorescence signal intensity values from manually segmented patched neurons. Patched cell numbers (Patched Cell ID) refer to the cells in Figure 3m. 


\section{Methods}

\section{Acute slice preparation}

Human cortical tissues were collected from adult patients undergoing neurosurgical procedures to treat symptoms associated with either epilepsy or brain tumor. Surgical specimens were obtained from local hospitals (Harborview Medical Center, Swedish Medical Center, and University of Washington Medical Center) in collaboration with local neurosurgeons. Surgically resected neocortical tissue was distal to the pathological core (i.e. tumor tissue or mesial temporal structures). Detailed histological assessment and using a curated panel of cellular marker antibodies indicated a lack of overt pathology in surgically resected cortical slices (Berg et al., 2020).

In this study, we included data from 15 surgical cases, 10 of which were epilepsy cases and the remaining 5 were tumor cases (49 \pm 15 years, mean \pm standard deviation, min: 21 , max: 68$)$. All specimens derived from neocortex with the majority of cases derived from the temporal lobe $(n=8)$ while a minority were obtained from the frontal lobe $(n=4)$ or parietal lobe $(n=1)$. A total of 12 acute slices were tested for the identification of gene expression with $\mathrm{mFISH} / \mathrm{HCR}$ after MPC recordings.

Surgical specimens were immediately transported (15-35 $\mathrm{min}$ ) from the operating room to the laboratory in chilled $\left(0-4^{\circ} \mathrm{C}\right)$ artificial cerebral spinal fluid (aCSF) slicing solution containing (in $\mathrm{mM}$ ): $92 \mathrm{~N}$-Methyl-D-glucamine (NMDG), $2.5 \mathrm{KCl}, 1.25 \mathrm{NaH}_{2} \mathrm{PO}_{4}, 30 \mathrm{NaHCO}_{3}, 20$ 4-(2-hydroxyethyl)-1-piperazineethanesulfonic acid (HEPES), 25 D-glucose, 2 thiourea, 5 Na-L-ascorbate, 3 Na-pyruvate, $0.5 \mathrm{CaCl}_{2}$, and $10 \mathrm{MgSO}_{4}$ (Ting et al., 2014). The NMDG aCSF was continuously bubbled with carbogen $\left(95 \% \mathrm{O}_{2}\right.$ and $\left.5 \% \mathrm{CO}_{2}\right)$. Osmolality was measured and adjusted to 300-315 mOsmoles/kg range (305-315 mOsmoles/kg range when using a freezing point osmometer, and 300-310 mOsmoles/kg range if using vapor pressure osmometer), and the $\mathrm{pH}$ was measured and adjusted to 7.3-7.4. $350 \mu \mathrm{m}$ thick human cortical slices were prepared using a Compressome VF-300 (Precisionary Instruments) or VT1200S (Leica Biosystems). After being cut, slices were transferred to oxygenated NMDG aCSF maintained at $34^{\circ} \mathrm{C}$ for $10 \mathrm{~min}$. Slices were kept at room temperature in oxygenated holding aCSF solution containing (in $\mathrm{mM}$ ): $92 \mathrm{NaCl}, 30 \mathrm{NaHCO}_{3}, 25 \mathrm{D}$-Glucose, $20 \mathrm{HEPES}, 5 \mathrm{Na}$-L-Ascorbate, 3 Na Pyruvate, $2.5 \mathrm{KCl}, 2 \mathrm{CaCl}_{2}, 2 \mathrm{MgSO}_{4}, 2$ Thiourea, $1.2 \mathrm{NaH}_{2} \mathrm{PO}_{4}$ prior to recording (Seeman et al., 2018; Berg et al., 2020).

\section{Slice culture preparation}

Following brain slice preparation and NMDG recovery steps as outlined above, a subset of brain slices were transferred to a 6-well plate for culture and viral transduction. Human cortical brain slices were placed on membrane inserts (Millipore \#PICMORG), and the wells were filled with $1 \mathrm{~mL}$ of culture medium consisting of $8.4 \mathrm{~g} / \mathrm{L}$ MEM Eagle medium, 20\% heat-inactivated horse serum, $30 \mathrm{mM}$ HEPES, $13 \mathrm{mM}$ D-glucose, $15 \mathrm{mM}$ $\mathrm{NaHCO}_{3}, 1 \mathrm{mM}$ ascorbic acid, $2 \mathrm{mM} \mathrm{MgSO}$, $1 \mathrm{mM} \mathrm{CaCl}$, $0.5 \mathrm{mM}$ GlutaMAX-I, and $1 \mathrm{mg} / \mathrm{L}$ insulin (Ting et al 2018). The slice culture medium was carefully adjusted to $\mathrm{pH} 7.2-7.3$, osmolality of $300-310 \mathrm{mOsmoles} / \mathrm{Kg}$ by addition of pure $\mathrm{H}_{2} \mathrm{O}$, sterile-filtered and stored at $4^{\circ} \mathrm{C}$ for up to two weeks. Culture plates were placed in a humidified $5 \% \mathrm{CO}_{2}$ incubator at $35^{\circ} \mathrm{C}$. 1-3 hours after brain slices were plated on cell culture inserts, brain slices were infected by direct application of concentrated AAV viral particles over the slice surface (Ting et al 2018). The slice culture medium was replaced every 2-3 days until initiating synaptic physiology experiments. The time window to perform slice culture experiments ranged from 2.5 to $7 \mathrm{DIV}$, and a total of 15 cultured human neocortical slices were used in this study for the identification of gene expression with mFISH/HCR after MPC recordings.

\section{Viral vector production}

Recombinant AAV vectors were produced by triple-transfection of ITR-containing enhancer plasmids along with AAV helper and rep/cap plasmids using the AAV293 cell line, followed by harvest, purification and concentration 
of the viral particles. The AAV293 packaging cell line and plasmid supplying the helper function are available from a commercial source (Cell Biolabs). The PHP.eB capsid variant was generated by Dr. Viviana Gradinaru at the California Institute of Technology (Chan et al., 2017) and the DNA plasmid for AAV packaging is available from Addgene (plasmid\#103005). Quality control of the packaged AAV was determined by qPCR to determine viral titer (viral genomes/mL), and by Sanger sequencing of the AAV genome to confirm the identity of the viral vector that was packaged.

\section{CN1390 vector design and construction}

Human neocortical interneurons were targeted in cultured slices by transducing slices with an optimized forebrain GABAergic viral vector CN1390, also known as pAAV-DLX2.0-SYFP2. The DLX 2.0 sequence includes a 3x concatemer of the core region of a previously well-characterized DLX I56i forebrain GABAergic neuron enhancer (Dimidischstein et al 2016; Zerucha et al, 2000). The $131 \mathrm{bp}$ core sequence of the hI56i enhancer was inferred from enhancer bashing experiments detailed in Zerucha et al, 2000. The $393 \mathrm{bp} 3 \mathrm{x}$ core enhancer concatemer sequence was custom gene synthesized and subcloned into pAAV-minBetaGlobin-SYFP2-WPRE3-BGHpA upstream of the minimal promoter to make pAAV-DLX2.0-SYFP2, vector ID\# CN1390 in our catalog. This vector will be deposited to Addgene for distribution to the academic community upon publication.

\section{Electrophysiology}

Experiments were conducted on an upright microscope with an oblique condenser (WI-OBCD, Olympus) equipped with infrared $(850 \mathrm{~nm})$ illumination, $490 \mathrm{~nm}, 565 \mathrm{~nm}$ and ultraviolet laser $(395 \mathrm{~nm})$ lines (Thorlab). 4x and 40x objectives (Olympus) were used to visualize the sample and a digital CMOS camera (Flash 4.0 V2, Hamamatsu) to take images. The rig configuration included eight electrodes disposed around the recording chamber, each surrounded by an headstage shield in order to prevent electrical crosstalk artifacts. Each patch electrode was positioned by xy stage and micromanipulator (PatchStar, Scientifica) with guidance of acq 4 open python platform software (acq4.org; Campagnola et al., 2014). Bright-field and fluorescent images were also captured and analyzed with acq4. Signals were amplified using Multiclamp 700B amplifiers (Molecular Devices) and digitized at 50-200 kHz using ITC-1600 digitizers (Heka). Pipette pressure was controlled using electropneumatic pressure control valves (Proportion-Air; PA2193). The recording software, Igor Pro7 or 8 (WaveMetrics), contained with a custom software Multi-channel Igor Electrophysiology Suite (MIES; https://github.com/AllenInstitute/MIES), used to apply the bias current, inject the appropriate amount of current to patched cells, data acquisition and pressure regulation.

Slices were transferred to the recording chamber and perfused with carbogenated aCSF ( $2 \mathrm{~mL} / \mathrm{min})$, constant temperature $\left(31-32{ }^{\circ} \mathrm{C}\right), \mathrm{pH}$ 7.2-7.3 and oxygen saturation in the recording chamber (40-50\%). Perfusing aCSF contained (in mM): $1.3 \mathrm{CaCl}_{2}, 12.5 \mathrm{D}$-Glucose, 1 or $2 \mathrm{MgSO}_{4}, 1.25 \mathrm{NaH}_{2} \mathrm{PO}_{4}, 3 \mathrm{KCl}, 18 \mathrm{NaHCO}, 126 \mathrm{NaCl}$, $0.16 \mathrm{Na}-\mathrm{L}-$ Ascorbate. Patch pipettes were pulled from thick-wall filamented borosilicate glass (Sutter Instruments) using a DMZ Zeitz-Puller (Zeitz) to a tip resistance of 3-8 M $\Omega$, and filled with intracellular solution containing (in mM) either 0.3 ethylene glycol-bis(b-aminoethyl ether)-N,N,N',N'-tetraacetic acid (EGTA) or no EGTA in addition to: $130 \mathrm{~K}$-gluconate, $10 \mathrm{HEPES}, 3 \mathrm{KCl}, 0.23 \mathrm{Na}_{2} \mathrm{GTP}, 6.35 \mathrm{Na}_{2}$ Phosphocreatine, $3.4 \mathrm{Mg}$-ATP, 13.4 Biocytin, and fluorescent dye with $50 \mu \mathrm{M}$ Alexa-488 or cascade blue. Solution osmolarity ranged from 280 to 295 mOsmoles/kg titrated with sucrose, $\mathrm{pH}$ between 7.2 and 7.3 titrated with $\mathrm{KOH}$. The liquid junction potentials were not corrected. For slice culture experiments, GABAergic neurons labeled with AAV-DLX2.0SYFP2 were targeted during patch pipettes were approaching. With cascade blue loaded in the patch pipette, overlaid signals in the same cells with both SYFP2 and cascade blue were confirmed by manual inspection of image stacks with blue and green LED light excitation.

Cell cluster of eight neurons at each trial was selected and attempt for multiple whole-cell patch-clamp (MPC) recordings, targeted in mainly supraganular layer (L2 and L3), 50-80 $\mu \mathrm{m}$ depth from slice surface and smooth 
somatic appearance. Pairwise recordings were performed for local synaptic connectivity assay with both voltage and current-clamp mode. In voltage-clamp mode, membrane voltages of all patched cells were hold at either -70 or $-55 \mathrm{mV}$ and brief depolarization to $0 \mathrm{mV}$ for $3 \mathrm{~ms}$ at $20 \mathrm{~Hz}$ sequentially to reliably identify both excitatory and inhibitory connections. In current-clamp mode, initially all cell membrane potentials were maintained at $-70 \pm 2$ $\mathrm{mV}$ with automated bias current injection when we generated presynaptic unitary action potential by brief current injections (1.5-3 ms) to detect EPSP responses in postsynaptic cells. For inhibitory connection, cell membrane potentials were maintained at $-55 \pm 2 \mathrm{mV}$ to detect IPSP responses in postsynaptic cells.

For the short-term plasticity, there are 12 action potentials at multiple frequencies $(10,20,50$, and $100 \mathrm{~Hz})$ to induce sequential postsynaptic responses in connected pairs. Presynaptic stimulus amplitudes were adjusted to generate unitary action potential in each pulse. In order to measure recovery time course after induction protocol (i.e. initial 8 pulses), inter-spike interval between $8^{\text {th }}$ and $9^{\text {th }}$ pulses at $50 \mathrm{~Hz}$ stimulation was varied sequentially at 125, 250, 500, 1000, and $4000 \mathrm{~ms}$. For other frequency stimulation (10, 20, and $100 \mathrm{~Hz})$, we used fixed 250 ms inter-spike interval between $8^{\text {th }}$ and $9^{\text {th }}$ pulses. Stimuli were interleaved between cells such that only one cell was spiking at a time, and no two cells were ever evoked to spike within $150 \mathrm{~ms}$ of each other (Seeman et al., 2018). At each sequential 12 pulses stimulation for all patched neurons were repeated with $15 \mathrm{~s}$ inter-sweep interval. After running connectivity protocol, step current injections in each cell were applied to extract intrinsic membrane properties such as spike shape and frequency-current relationship.

\section{Data analysis}

Synaptic connectivity and dynamics, intrinsic membrane properties were analyzed with custom-written MATLAB (MathWorks) and Igor (Wavemetrics) software. Somatic position of individual neurons in a cluster from electrophysiological recording was imaged with fluorescent dyes (Alexa488 or cascade blue) with upright microscope and saved in ACQ4. Consequently, recorded neurons were identified with biocytin staining image and matched with $\mathrm{mFISH/HCR} \mathrm{signals} \mathrm{taken} \mathrm{by} \mathrm{inverted} \mathrm{confocal} \mathrm{microscope.}$

\section{Thick tissue mFISH sample preparation}

Slices were fixed in 4\% PFA for 2 hours at room temperature (RT), washed three times in PBS for 10 min each, then transferred to $70 \% \mathrm{EtOH}$ at $4^{\circ} \mathrm{C}$ for a minimum of 12 hours, and up to 30 days. Slices were then incubated in $8 \%$ SDS in PBS at RT for two hours with agitation. The solution was exchanged with $2 \mathrm{X}$ sodium chloride sodium citrate (SSC) three times, slices were washed for one hour at RT, followed by two additional (1 hour each) washes with fresh 2X SSC.

\section{In situ HCR for thick tissue}

We performed HCR v3.0 using reagents and a modified protocol from Molecular Technologies and Molecular Instruments (Choi et al., 2014). Slices were incubated in pre-warmed 30\% probe hybridization buffer (30\% formamide, $5 \mathrm{X}$ sodium chloride sodium citrate (SSC), $9 \mathrm{mM}$ citric acid $\mathrm{pH} 6.0,0.1 \%$ Tween $20,50 \mu \mathrm{g} / \mathrm{mL}$ heparin, 1X Denhardt's solution, $10 \%$ dextran sulfate) at $37^{\circ} \mathrm{C}$ for $5 \mathrm{~min}$, then incubated overnight at $37^{\circ} \mathrm{C}$ in hybridization buffer with the first three pairs of probes added at a concentration of $4 \mathrm{nM}$. The hybridization solution was exchanged 3 times with $30 \%$ probe wash buffer (30\% formamide, 5X SSC, 9 mM citric acid pH 6.0, $0.1 \%$ Tween $20,50 \mu \mathrm{g} / \mathrm{mL}$ heparin) and slices were washed for one hour at $37^{\circ} \mathrm{C}$. Probe wash buffer was briefly exchanged with 2X SSC, then amplification buffer (5X SSC, 0.1\% Tween 20, 10\% dextran sulfate) for 5 min. Even and odd hairpins for each of the three genes were pooled and snap-cooled by heating to $95^{\circ} \mathrm{C}$ for 90 seconds then cooling to RT for $30 \mathrm{~min}$. The hairpins were then added to amplification buffer at a final concentration of 60 $\mathrm{nM}$, and slices were incubated in amplification solution for 4 hours at RT. This was followed by a brief wash with 2X SSC and a one hour, room temperature incubation in $2 \mathrm{X}$ SSC containing $8 \mu \mathrm{g} / \mu 1$ Brilliant Violet 421TM Streptavidin (BioLegend, Cat. No. 405225) and 0.05\% Tween 20. Slices were washed three times for 10 min in $2 \mathrm{X}$ SSC. For each round of imaging, an aliquot of $67 \%$ 2,2'-Thiodiethanol (TDE) solution was prepared for use 
as a clearing and immersion fluid. $\geq 99 \%$ TDE (Sigma-Aldrich) was mixed with DI water to create a $67 \%$ TDE solution with a refractive index of 1.46, verified by a pocket refractometer (PAL-RI, Atago). Slices were transferred to $67 \% \mathrm{TDE}$ and allowed to equilibrate for at least 1 hour at room temperature prior to imaging.

\section{Quantification of thick tissue mFISH signals}

Patched cells from acute and cultured tissues were hand segmented volumetrically using QuPath software (Bankhead et al., 2017). Segmentation was performed on either the SYFP2 labeled cell body (slice culture preparation) or HCR signal (acute slice preparation) in transcript positive cells. Additionally, several nearby cells were also segmented in order to characterize typical expression levels in each probed gene and to compare signal level to patched cells. For each imaged channel, a histogram of non-cellular pixels was used to calculate a background threshold, which was taken to be three times the half width at half maximum above median of the distribution of pixel values. A mask of lipofuscin pixels was constructed by first taking all pixels that exceeded this threshold in all HCR channels. This mask was additionally expanded by morphological dilation with a kernel of radius one pixel, iterated two times. For each segmented cell, this mask was applied to each channel and the remaining intensity above background was integrated and normalized to the cell volume, this is taken as a measure of expression in each channel and reported in Figure 3 and Extended Data Figures 3,5,6.

\section{Confocal imaging}

Thick tissue images were acquired on an Olympus FV3000 confocal microscope using a 30X silicon oil objective with the zoom set to $1.5 \mathrm{x}$. The image montage stacks were acquired through the depth of the tissue at $1.2 \mu \mathrm{m}$ steps. For figures, maximum intensity projections though the region of interest were generated are shown. Note that some montages exhibit stitching artifacts. Due to the frequent appearance of lipofuscin in aging human tissues, we showed HCR images as multiple overlapping channels since the lipofuscin granules were revealed as spots that are fluorescent in every channel.

\section{Stripping and subsequent hybridization rounds}

To strip the signal in preparation for subsequent rounds, 67\% TDE was exchanged with 2X SSC three times and samples were washed for 1 hour. 2X SSC was replaced with $1 \mathrm{X}$ DNase buffer for $5 \mathrm{~min}$ and then a 1:50 dilution of DNase I in DNase buffer (DNase I recombinant, RNase-free, Roche, Cat. No. 04716728001), and incubated for 1 hour at $37^{\circ} \mathrm{C}$. This solution was replaced with fresh DNase solution before incubating slices overnight at $37^{\circ} \mathrm{C}$. Slices were washed with $65 \%$ formamide in $2 \mathrm{X} \mathrm{SSC}$ for one hour at $37^{\circ} \mathrm{C}$, then $2 \mathrm{X} \mathrm{SSC}$ for one hour at $\mathrm{RT}$, before being transferred to $67 \%$ TDE for at least one hour. After imaging to confirm the signal was gone, the slices were washed in $2 \mathrm{X} \mathrm{SSC}$ for one hour to remove TDE before proceeding to subsequent hybridization rounds, which followed the protocol described above, except omitting the incubation in streptavidin solution.

\section{Thin tissue section mFISH sample preparation}

Human neocortical tissue from surgical resections was frozen in OCT without fixation. 10 $\mu \mathrm{m}$ sections were cut and attached to silanized $\# 1.5$ coverslips and stored in a sealed chamber at $-80^{\circ} \mathrm{C}$. For staining, samples were thawed in fresh 4\% PFA for 15 minutes, and then transferred to 70\% ethanol and incubated for either 2 hours or overnight. Permeabilize for 10 minutes in $8 \%$ SDS in 1xPBS at room temperature, then wash in $70 \%$ ethanol twice with one-minute incubations, and dry slide completely. SecureSeal Hyb chambers (Grace Bio-Labs) were adhered to the coverslip for staining.

\section{In situ HCR for thin sections}

HCR was conducted as in thick tissues, with the exception that the probe wash time was reduced to 30 minutes, and HCR hairpin amplification was reduced to 1 hour at room temperature. Stripping the HCR signal for multiround labeling was carried out in a similar manner to thick tissue sections, but with only a single incubation for two hours in DNase1 solution (Extended Data Fig. 2). 


\section{Thin section imaging and analysis}

After HCR amplification, anti-photobleaching buffer (1mM Trolox solution (Sigma Aldrich), 40U/ml Catalase (Abcam), 40U/ml Glucose oxidase (Sigma Aldrich), and 1:100 RNase inhibitor (Clontech) was applied to stained samples in SecureSeal chambers on coverslips. The coverslips were placed in a stainless steel chamber fitted to the insert of the motorized $x-y$ stage (ASI MS-2000). Samples were imaged on an inverted microscope (Zeiss AxioObserver) with a $63 \mathrm{x}$ oil immersion objective in the epifluorescence configuration with a square fiber laser illumination system (Andor Borealis). Each round of imaging produced four fluorescence channels (DAPI, Alexa 488, Alexa 568, Alexa 647) in z stacks at a multiple fields of view (FOV), where each FOV is the same nominal $(\mathrm{x}, \mathrm{y})$ location in each round of imaging. FOV images from each imaging round were registered to the first round using intensity correlation of the lipofuscin autofluorescence visible in the $\mathrm{z}$-stack maximum projection of the 488 channel. In each FOV, HCR puncta were localized using bandpass filtering and local-max-peak finding, yielding HCR spot locations with their integrated brightness and radius of gyration. Lipofuscin autofluorescence was removed from this HCR signal by filtering the large and bright particles. Further lipofuscin rejection was accomplished by eliminating particles found within a 2-pixel $(400 \mathrm{~nm})$ radius in all channels in any imaging round. The remaining HCR signal was associated with individual inhibitory cells following manual segmentation of GAD1-positive cell bodies in FIJI. The 421 inhibitory cells in Extended Data Figure 2, were grouped in the heat map based on a binary thresholds on HCR spots per cell area.

\section{Morphological reconstruction}

Reconstructions of the dendrites and the initial part of the axon (spiny neurons) and/or the full axon (aspiny/sparsely spiny neurons) were generated for a subset of neurons with good-quality electrophysiology and biocytin fills. Reconstructions were generated based on a 3D image stack taken by confocal microscope that was run through a Vaa3D-based image processing and reconstruction pipeline (Peng et al., 2010). The process could include a variable enhancement of the signal-to-noise ratio in the image (Peng et al., 2014). Reconstructions were manually corrected and curated using a range of tools (e.g., virtual finger, polyline) in the Mozak extension (Zoran Popovic, Center for Game Science, University of Washington) of Terafly tools (Peng et al., 2014; Bria et al., 2016) in Vaa3D. Every attempt was made to generate a completely connected neuronal structure while remaining faithful to image data. If axonal processes could not be traced back to the main structure of the neuron, they were left unconnected. As a final step in the manual correction and curation process, an alternative analyst checked for missed branches or inappropriate connections. Once the reconstruction was deemed complete, multiple plugins were used to prepare neurons (saved as SWC files) for morphological analyses.

\section{References for Methods}

Bankhead, P. et al. QuPath: Open source software for digital pathology image analysis. Sci Rep 7, 16878. doi: 10.1038/s41598-017-17204-5 (2017).

Bria, A. et al. TeraFly: real-time three-dimensional visualization and annotation of terabytes of multidimensional volumetric images. Nat. Methods 13, 192-194 (2016).

Campagnola, L. et al. ACQ4: an open-source software platform for data acquisition and analysis in neurophysiology research. Front Neuroinform 8, 3. doi: 10.3389/fninf.2014.00003 (2014).

Chan, K.Y. et al. Engineered AAVs for efficient noninvasive gene delivery to the central and peripheral nervous systems. Nat Neurosci. 20, 1172-1179 (2017).

Choi, H.M. et al. Next-generation in situ hybridization chain reaction: higher gain, lower cost, greater durability. ACS Nano 8, 4284-4294 (2014).

Peng, H. et al. V3D enables real-time 3D visualization and quantitative analysis of large-scale biological image data sets. Nat. Biotechnol. 28, 348-353 (2010). 
663

664

665

666

667

668

669

670

671

672

673

674

675

676

677

678

679

680

681

682

683

684

685

686

687

688

689

690

691

692

693

694

695

Peng, H. et al. Extensible visualization and analysis for multidimensional images using Vaa3D. Nat Protoc 9, 193-208 (2014).

Ting, J.T. et al. Acute brain slice methods for adult and aging animals: application of targeted patch clamp analysis and optogenetics. Methods Mol. Biol. 1183, 221-242 (2014).

Zerucha, T. et al. A highly conserved enhancer in the Dlx5/Dlx6 intergenic region is the site of cross-regulatory interactions between Dlx genes in the embryonic forebrain. J. Neurosci. 20, 709-721 (2000).

\section{Acknowledgments}

We thank the Tissue Procurement, Tissue Processing, and Facilities teams for human tissue collection and brain slice preparation. We thank the hospital coordinators that help with logistics of collections and patient consent. We thank the Viral Technology team for packaging AAV vectors. We thank Lydia Potekhina and Shea Ransford for helping imaging on the confocal microscope. We thank Jennie Close for providing thin tissue sections from human donors. We thank Dr. Viviana Gradinaru for the gift of PHP.eB capsid packaging plasmid. We thank Dr. Christof Koch for comments on the manuscript. This work is supported in part by NIH BRAIN Initiative award \#1RF1MH114126-01 from the National Institute of Mental Health to E.S.L., J.T.T., and B.P.L. The content is solely the responsibility of the authors and does not necessarily represent the views of the funding agencies. In addition, we wish to thank the Allen Institute for Brain Science founder, Paul G. Allen, for his vision, encouragement and support.

\section{Author contributions}

M.H.K., J.T.T., B.P.L., E.S.L. conceptualized the project. N.D., A.L.K., J.G.O., D.L.S., R.P.G., C.C., C.D.K. procured human surgical tissues for the project. M.H.K., C.R. performed MPC recording experiments. L.C., T.J. provided technical support for MPC recordings. E.R.T., J.T.M. performed post-hoc mFISH staining and imaging with guidance of B.P.L. S.K., C.G. reconstructed morphologies of patched neurons with guidance of S.A.S. J.T.T. developed the DLX2.0 enhancer and CN1390 AAV vector and performed human ex vivo slice culture and viral transduction. B.L., M.J.T. performed quantification of HCR signals with guidance of P.R.N. M.H.K., T.J., G.M., F.D. provided synaptic physiology project leadership and budgetary management. H.Z., E.S.L. provided cell type program leadership. M.H.K., C.R., E.R.T., B.L., M.J.T., B.P.L. analyzed data and prepared the figures. M.H.K., C.R., J.T.T., B.P.L., E.S.L. wrote the manuscript with input from all authors.

\section{Competing financial interests}

J.T.T., B.P.L, E.S.L are inventors on U.S. patent application \#PCT_US2019_054539 related to this work (vector CN1390). All authors declare no other competing interests. 